INPLASY

PROTOCOL

To cite: He et al. Effects of acupuncture on neuropathic pain induced by spinal cord injury: a systematic review and meta-analysis. Inplasy protocol 2021100107. doi:

10.37766/inplasy2021.10.0107

Received: 27 October 2021

Published: 27 October 2021

Corresponding author:

Ruijie Ma

maria7878@sina.com

Author Affiliation:

Zhejiang Chinese Medical

University

Support: Ruijie Ma.

Review Stage at time of this submission: Data analysis.

Conflicts of interest:

None declared.

\section{Effects of acupuncture on neuropathic pain induced by spinal cord injury: a systematic review and meta-analysis}

\author{
He, K¹; Hu, R²; Qiu, B³ Huang, Y4; Chen, Q5; Ma, R6.
}

Review question / Objective: This study aimed to conduct a systematically review and meta-analysis to the efficacy of acupuncture on neuropathic pain induced by spinal cord injury.

Condition being studied: Neuropathic pain is a common consequence and the most intractable comorbidities following spinal cord injury (SCl), which compromises a person's life satisfaction and quality. Currently, there are limited available treatments for $\mathrm{SCl}$-induced neuropathic pain in clinical practice. Acupuncture as an integral part of traditional medicines throughout the world to management acute and chronic pain has recently drawn widespread attention and increasingly applied in clinical and scientific research.

INPLASY registration number: This protocol was registered with the International Platform of Registered Systematic Review and Meta-Analysis Protocols (INPLASY) on 27 October 2021 and was last updated on 27 October 2021 (registration number INPLASY2021100107).

\section{INTRODUCTION}

Review question / Objective: This study aimed to conduct a systematically review and meta-analysis to the efficacy of acupuncture on neuropathic pain induced by spinal cord injury.
Condition being studied: Neuropathic pain is a common consequence and the most intractable comorbidities following spinal cord injury ( $\mathrm{SCl})$, which compromises a person's life satisfaction and quality. Currently, there are limited available treatments for SCl-induced neuropathic pain in clinical practice. Acupuncture as an 
integral part of traditional medicines throughout the world to management acute and chronic pain has recently drawn widespread attention and increasingly applied in clinical and scientific research.

\section{METHODS}

Participant or population: Adult participants (>18years of age) diagnosed as $\mathrm{SCl}$-induced neuropathic pain.

Intervention: The intervention in the acupuncture group included acupuncture alone or in combination with conventional therapy.

Comparator: The control group included conventional therapy and/or sham acupuncture.

Study designs to be included: Published randomized controlled trials.

Eligibility criteria: This study will included all published randomized controlled trials (RCTs). Any other studies, such as review, animal studies, case reports, case series, letters, comments, non-clinical trials, nonRCTs, and quasi-RCTs studies were all excluded.

Information sources: Pubmed, Cochrane library, Web of science, CNKI, CBM, Wanfang database and VIP.

Main outcome(s): Pain severity.

Additional outcome(s): Present Pain Intensity and Pain Region Index.

Quality assessment / Risk of bias analysis: Cochrane risk of bias assessment tool.

Strategy of data synthesis: Continuous data was calculated as mean differences (MD) and $95 \%$ confidence interval (CI). If the measurements of MD varied between studies, standardised MD (SMD) was calculated for meta-analysis.

Subgroup analysis: Subgroup analysis were carried out according to different acupuncture methods.
Sensitivity analysis: sensitivity analyses were carried out to dissect the heterogeneity

Country(ies) involved: China.

Keywords: acupuncture, spinal cord injury, neuropathic pain, meta-analysis, systematic review

Contributions of each author:

Author 1 - Kelin He.

Email: 352128492@qq.com

Author 2 - Rong Hu.

Email:m18768156037@163.com

Author 3 - Bei Qiu.

Email: 1109476936@qq.com

Author 4 - Yi Huang.

Email: hyhaha0212@163.com

Author 5 - Qinqin Chen.

Email: 1019456432@qq.com

Author 6 - Ruijie Ma.

Email: maria7878@sina.com 